\title{
Calculer le salaire horaire avant de comparer les revenus
}

\section{Christoph Bosshard}

Dr méd., vice-président de la FMH, responsable du département Données, démographie et qualité

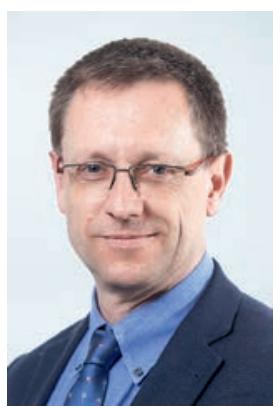

A la FMH, la transparence sur les revenus des médecins est une longue tradition: pendant des décennies, notre association a publié chaque année une enquête sur les revenus dans le Bulletin des médecins suisses. Puis en 2013, suite à une modification du cadre juridique, l'Office fédéral des assurances sociales (OFAS) a interdit l'acquisition des données des caisses de compensation sur les revenus soumis à l'AVS nécessaires aux calculs. L'OFAS estimait en outre que l'utilisation des revenus soumis à l'AVS ne permettait pas de tirer des conclusions suffisamment probantes sur les revenus réels, ce que la FMH avait elle aussi toujours déploré.

Nous nous sommes alors demandés comment continuer. L’approche du relevé MAS, effectué par l'Office fédéral de la statistique (OFS), nous a finalement semblé très prometteuse. La FMH pouvait compter sur le grand

La FMH a commandé une étude pour éclaircir la question du salaire horaire et disposer ainsi d'un critère de comparaison nuancé.

professionnalisme de ce partenaire dans la collecte et le traitement des données. Portant sur les données de 2015, la première enquête a été achevée à l'été 2017 et peut être considérée comme une réussite à tous égards. Le taux de participation élevé a permis de réaliser les analyses statistiques souhaitées.

L'utilisation des revenus soumis à l'AVS a également pour inconvénient que les chiffres ne font pas ressortir la part du revenu dégagée de l'activité médicale. En revanche, les données du relevé MAS renseignent de manière nuancée sur le produit et les charges (taux de coûts) d'un cabinet, et donc sur le revenu d'un médecin indépendant. La FMH ne conteste pas l'intérêt public prépondérant dans le domaine des revenus basés sur les assurances sociales. Les chiffres du relevé MAS indiquent désormais quelles composantes salariales sont générées par les assurances sociales, quelle somme provient des assurances complémentaires et quels revenus sont générés par d'autres activités médicales, telles que des expertises. En effet, les médecins restent libres de gagner de l'argent en dehors du système de santé.

Selon les chiffres du relevé MAS publiés le 10 avril 2018, le résultat d'exploitation moyen d'un cabinet individuel était de 155000 francs en 2015, la part issue des assurances sociales s'élevant à 138000 francs. En moyenne suisse, il s'agit de revenus attrayants. Or, avant de faire des comparaisons, il faut répondre à plusieurs questions: combien d'heures de travail sont nécessaires pour générer ces revenus? Dans quelle phase de la vie et pendant combien de temps peut-on fournir un tel travail? La FMH a voulu clarifier les choses en abordant la question du salaire horaire.

Je tiens à remercier les auteurs de l'étude présentée dans ce numéro, qui contribue aussi à améliorer la transparence. Cette analyse, également basée sur un relevé de l'OFS, aboutit à un résultat très clair: le corps médical ne monte pas sur le podium, mais, avec un salaire horaire de 67 francs, termine à la $4^{\mathrm{e}}$ place derrière les dentistes, les avocats et les économistes. En conclusion, le revenu annuel des médecins relativement élevé par rapport à d'autres groupes professionnels s'explique par des horaires de travail supérieurs à la moyenne. Il est parfois nécessaire d'y regarder de plus près pour obtenir des comparaisons nuancées. La FMH continuera de s'investir en faveur de la transparence

\section{Le revenu annuel relativement élevé des} médecins s'explique par des horaires de travail supérieurs à la moyenne.

et de comparaisons équitables! Afin que les médecins, mais aussi les milieux politiques et l'administration publique, puissent disposer d'une base de données fiable et solide, le Comité central de la FMH recommande aux membres, aux cabinets médicaux et aux centres ambulatoires de participer à l'enquête MAS 2017 menée actuellement par l'Office fédéral de la statistique. 